Journal of Mathematics and Statistics 5 (2):90-92, 2009

ISSN 1549-3644

(C) 2009 Science Publications

\title{
Application of Sivasubramanian Kalimuthu Hypothesis to Triangles
}

\author{
M. Sivasubramanian \\ Department of Mathematics, Dr. Mahalingam College of Engineering and Tec ogy, \\ Polachi, Tamilnadu-642003, India
}

\begin{abstract}
Problem statement: The interior angles sum of a number of Ey
transformed into quadratic equations. The analysis of those quadratic equatio proposition: There exists Euclidean triangle whose interior angle sum is a st this study, the researchers introduced a new hypothesis for quadratic equ new result. Results: The result of the study was controversial, establish the quadratic equation's hypothesis.
\end{abstract}

Key words: Quadratic equations, tachyon physics, Euclid,

INTRODUCTION

For two thousand years, many attempts were made to prove the parallel postulate using Euclid's first fo postulates. The main reason that such a proof wa highly sought after was that the fifth postulate isn't sc evident unlike the other postulates. If the order th postulates were listed in the Elements is significant, it indicates that Euclid included this posty he realised he could not prove it or pr

Ibn Al-Haytham (Alhazen) mathematician, made the first parallel postulate using a pronf by he introduced the concept $c$ into geometry. He sformation quadrilateral, which Abramovich nfeld names the "Ibn al-y a bert quadrilat al and his attempted proo a so sho, ilarities to Playfair's axiom.

Omar $Y$ Jám (1050-1123) ì

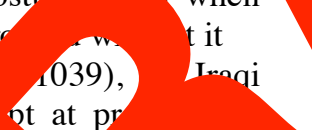
inter: nd it is impo for two convergent straight lines a rge in the $g$ tion in which they converge. $\mathrm{He}$ rec that ee possibilities arose from omitting b , if two perpendiculars to one cross another 1 ine, judicious choice of the last can in internal angles where it meets the two pery equal (it is then parallel to the first line). if a equal internal angles are right angles, we get Iid's Fifth; otherwise, they must be either acute or Ise. He persuaded himself that the acute and obtuse o lead to contradiction, but had made a tacit assumption equivalent to the fifth to get there.

Nasir al-Din al-Tusi (1201-1274), in his Al-risala al-shafiya'an al-shakk fi'l-khutut al-mutawaziya (Discussion Which Removes Doubt about Parallel Lines) (1250), wrote detailed critiques of the parallel postulate and on Khayyám's attempted proof a century earlier. Nasir al-Din attempted to derive a proof by contradiction of the parallel postulate $\mathrm{He}$ was also one of the first to consider the cases of elliptical geometry and hyperbolic geometry, though he ruled out both of them.

Euclidean, elliptical and hyperbolic geometry. The Parallel Postulate is satisfied only for models of Euclidean geometry.

Nasir al-Din's son, Sadr al-Din (sometimes known as "Pseudo-Tusi"), wrote a book on the subject in 1298, based on Nasir al-Din's later thoughts, which presented one of the earliest arguments for a non-Euclidean hypothesis equivalent to the parallel postulate. "He essentially revised both the Euclidean system of axioms and postulates and the proofs of many propositions from

Corresponding Author: M. Sivasubramanian, Department of Mathematics, Dr. MahalinGam College of Engineering and Technology, Polachi, Tamilnadu-642003, India 
the elements. His research was published in Rome in 1594 and was studied by European geometers. This study marked the starting point for Saccheri's work on the subject.

Giordano Vitale (1633-1711), in his book Euclide restituo $(1680,1686)$, used the Khayyam-Saccheri quadrilateral to prove that if three points are equidistant on the base $\mathrm{AB}$ and the summit $\mathrm{CD}$, then $\mathrm{AB}$ and $\mathrm{CD}$ are everywhere equidistant. Girolamo_Saccheri (16671733) pursued the same line of reasoning more thoroughly, correctly obtaining absurdity from the obtuse case (proceeding, like Euclid, from the implicit assumption that lines can be extended indefinitely and have infinite length), but failing to debunk the acute case (although he managed to wrongly persuade himself that he had).

Where Khayyám and Saccheri had attempted to prove Euclid's fifth by disproving the only possible alternatives, the nineteenth century finally saw mathematicians exploring those alternatives and discovering the logically_consistent geometries which result. In 1829, Nikolai Ivanovich Lobachevsky published an account of acute geometry in an obsc Russian journal (later re-published in 1840 in Germ In 1831, János_Bolyai included, in a book by his fath an appendix describing acute geometry, which doubtlessly, he had developed independently of Lobachevsky. Carl Friedrich Gauss had tudied the problem before that, but he did abl of his results. However, upon hearing oylai's in a letter from Bolyai's father, Fari

"If I commenced by saning praise this study, you wou' moment. But I cannot s? erwise. To it would be to praise myself. the whole con of the work, the path take ne the results which he is led, coincide rost en. vith my meditations, which have of ied my mind, for the last thirty or thirty-fiy rs."

The ang ge netries were lân developed by Lobac sy, Rie 1 and Poincaré into hyperbolic geome. case) a spherical geometry (the obtuse cas Indepen of the parallel postulate

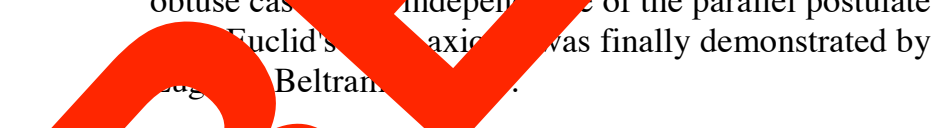

Con In the Euclidean construction as shown in

Draw a ngle $\mathrm{ABC}$. Choose points D and $\mathrm{E}$ on Join A and D; Join A and E.

vall letters $\mathrm{x}, \mathrm{y}$ and $\mathrm{z}$ denote the sum of the into angles of triangles $\mathrm{ABD}, \mathrm{ADE}$ and $\mathrm{AEC}$ respecrively. Let $\mathrm{a}, \mathrm{b}$ and $\mathrm{c}$ respectively refer to the sum of the interior angles in triangles $\mathrm{ABE}, \mathrm{ADC}$ and $\mathrm{ABC}$.

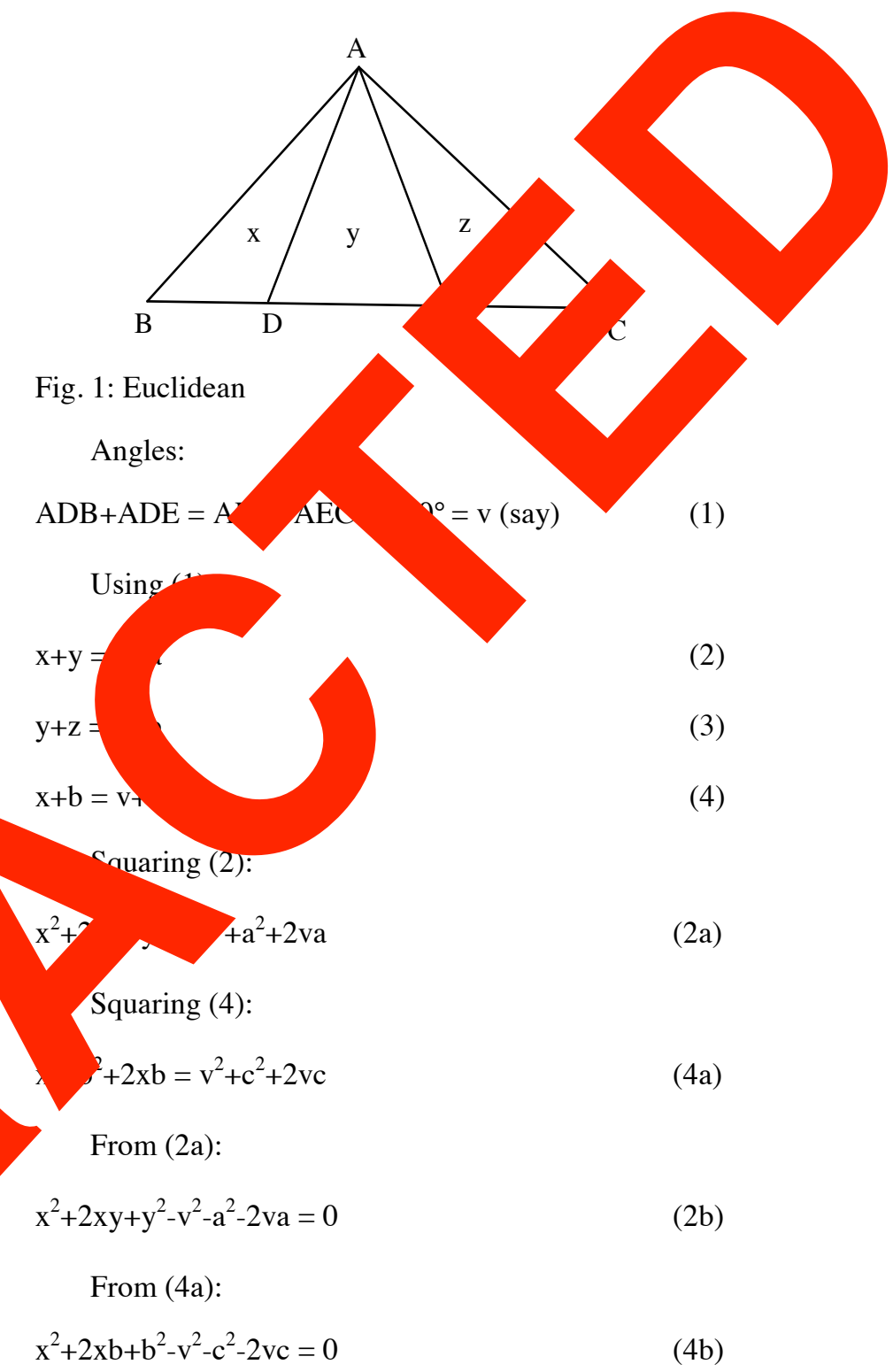

If $\alpha$ and $\beta$ are the roots of (2b), then according to the laws of quadratic equations,

$\alpha+\beta=-\frac{\mathrm{B}}{\mathrm{A}}$

and

$\alpha \beta=\frac{\mathrm{C}}{\mathrm{A}}$

(5)+(5a) shows:

$\alpha+\beta+\alpha \beta=\frac{C-B}{A}$ 
Applying (6) in (2b):

$\alpha+\beta+\alpha \beta=-2 y-v^{2}-a^{2}-2 v a$

Assuming (6) in (4b):

$\alpha+\beta+\alpha \beta=-2 b-v^{2}-c^{2}-2 v c$

Applying Sivasubramanian Kalimuthu hypothesis in (7) and (8):

$2 \mathrm{y}+\mathrm{v}^{2}+\mathrm{a}^{2}+2 \mathrm{va}=2 \mathrm{~b}+\mathrm{v}^{2}+\mathrm{c}^{2}+2 \mathrm{vc}$

i.e., $2 b-2 y+c^{2}-a^{2}+2 v(c-a)=0$

$2 \mathrm{~b}-2 \mathrm{y}+(\mathrm{c}+\mathrm{a})(\mathrm{c}-\mathrm{a})+2 \mathrm{v}(\mathrm{c}-\mathrm{a})=0$

i.e., $2 b-2 y+(c-a)(c+a+2 v)=0$

(4)-(2) gives $b-y=c-a$

Putting (10) in (9), $2(\mathrm{c}-\mathrm{a})+(\mathrm{c}-\mathrm{a})(\mathrm{c}+\mathrm{a}+2 \mathrm{v})=0$

$$
\text { i.e., }(c-a)(2 v+2+c+a)=0
$$
$\mathrm{c}-\mathrm{a}=0$ :

Since the second factor can NOT be equal to i.e., $\mathrm{c}=\mathrm{a}$

Putting (11) in (10): $y=b$

\section{Applying (12) in (3).} $\mathrm{z}=\mathrm{v}=180^{\circ}[$ by 1$]$

From (13) get that the $\mathrm{s}$ of triangle:

$\mathrm{AEC}$ ic aight

\section{RIALS METHODS}

ition multiplication and division are th our fundar al operations of number theory. Mul is the shortest form of addition. And di in is est way of subtraction. So, these four ations aro reduced to only two operations viz. tion and subtraction. By applying the "addition" $o_{1} n$ of number theory, the triangle properties were trans med into linear algebraic equations and the linear algebraic equations were converted into quadratic equations. The laws of set theory may be appl future investigations.

\section{RESULTS}

Although the result (14), i.e. interior angles of the Euclidean ty right angle is controversial, consistent. This result can be Lobachevsky, Riemann triangles

Let us recall Legendre shown at if the $f$ the interior angles of any triangle is equal to two ngles, the parallel postulate ince we have ed (14) without assum ale fith Euclidean posturate, Eq. 14 proves the if lel postulate ${ }^{\left[1-3^{3}\right)}$ But the mere existence of cons models Non-Euclidean geometries demo the Eucl cannot be deduced from Euclid $\mathrm{dIV}$. B r result is consistent. There is something sure of mathematics. Further he will unloun wis mystery.

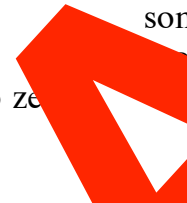

(11) quation 14 reveals that there is something hidden sures of mathematics. Only further studies will

$\mathrm{k}$ this mystery. Future probe may give rise to a $\mathrm{n}, \mathrm{w}$ field of geometry.

\section{ACKNOWLEDGEMENT}

The researcher wishes to thank the management of Dr. Mahalingam College of Engineering and Technology, Polachi, Tamilnadu-642003, India for their kind encouragement for the preparation of this research.

\section{REFERENCES}

1. Effimov, N.V., 1972. Higher Geometry. 1st Edn., Mir Publishers, Moscow, pp: 1-30.

2. Sivasubramanian, M. and S. Kalimuthu, 2008. On the new branch of mathematical science. J. Math.

Stat., 4:

122-123. http://www.scipub.org/fulltext/jms2/jms242122123.pdf

3. Sivasubramanian, M. and S. Kalimuthu, 2008. On the new branch of mathematical science part 2. J. Math. Stat., 4: 148-149. http://www.scipub.org/fulltext/jms2/jms243148149.pdf 